\title{
The Osteogenic Potential of Human Non- differentiated and Pre-differentiated Mesenchymal Stem Cells Combined with an Osteoconductive Scaffold - Early Stage Healing
}

\author{
Luboš Tuček¹, Zuzana Kočí2,3,4, Kristýna Kárová2,3, Helena Doležalová1, Jakub Suchánek ${ }^{1, *}$
}

\begin{abstract}
Despite the huge research into stem cells and their regenerative properties for bone healing, there are still unanswered questions including the recipient's respond to the presence of the stem cells, the fate of stem cells inside the bone defect and the possible advantage in utilizing pre-differentiated cells. To address these problems, we used human multipotent mesenchymal stromal/stem cells (MSCs), GMP Grade, in a rat model of bone formation. In a "bioreactor concept" approach seven Wistar rats were implanted with $0.2 \mathrm{~g}$ of synthetic bone scaffold seeded with $2 \times 106$ MSCs, seven Wistar rats were implanted with $0.2 \mathrm{~g}$ of synthetic bone scaffold seeded with $1 \times 106$ predifferentiated osteoblasts and $1 \times 106$ pre-differentiated endothelial cells and 14 Wistar rats were implanted with $0.2 \mathrm{~g}$ of synthetic bone scaffold without seeded cells into an intramuscular pocket on the left side of their back. The right side of each rat was used as a control, and $0.2 \mathrm{~g}$ of synthetic bone scaffold was implanted into the intramuscular pocket alone. To see the early stage healing the samples were harvested 14 days after the implantation, MSCs were detected by positive DAPI and MTCO2 staining in 43\% of all the samples implanted with MSCs, and no inflammation signs were present in any implanted animal. New vessels could be found in both groups implanted with MSCs, but not in the control group of animals. However, hematoxylin-eosin staining could not detect newly created bone within the implant in any of the groups. These results were in line with COLL1 staining, where we could detect positive staining only in three cases, all of which were implanted with un-differentiated MSCs. According to our findings, there were no benefits of using the pre-differentiated of MSC.
\end{abstract}

\section{KEYWORDS}

mesenchymal stromal/stem cells; bone defect; osteoconduction; osteoinduction; scaffold; tissue bone engineering

\author{
AUTHOR AFFILIATIONS \\ ${ }^{1}$ Department of Dentistry, Charles University, Medical Faculty and University Hospital Hradec Králové, Czech Republic \\ ${ }^{2}$ Institute of Experimental Medicine ASCR, Prague, Czech Republic \\ ${ }^{3}$ Department of Neuroscience, 2nd Faculty of Medicine, Charles University, Czech Republic \\ ${ }^{4}$ Bioinova, Ltd, Prague, Czech Republic \\ * Corresponding author: University Hospital Hradec Králové, Department of Dentistry, Sokolská 581, 50005 Hradec Králové, \\ Czech Republic; e-mail: suchanekj@lfhk.cuni.cz
}

Received: 25 November 2016

Accepted: 20 December 2016

Published online: 6 March 2017

Acta Medica (Hradec Králové) 2017; 60(1): 12-18

https://doi.org/10.14712/18059694.2017.43

(c) 2017 The Authors. This is an open-access article distributed under the terms of the Creative Commons Attribution License (http://creativecommons.org/licenses/by/4.0), which permits unrestricted use, distribution, and reproduction in any medium, provided the original author and source are credited. 


\section{INTRODUCTION}

\section{MANAGEMENT OF THE BONE DEFECTS}

Bone disorders connected with the aging population, low physical activity and higher body weight starts to represents challenging tasks for nowadays and mostly close future medicine. Most commonly found disorders are bone cysts, benign or malign tumors and pathological fracture. The first two mentioned represent diseases characterized by a silent slow progression and accompanied with wide bone destruction. The result of the surgery and its efficiency is mostly based on the size of the bone defect. The small defect can be left without any treatment and undergo physiological regeneration similar way to the healing of a bone fracture. This standard procedure works very well in case of small defects but larger defects are often accompanied by some complications, such as pathological fracture or dehiscence of the wound with secondary infection and subsequently blood clot disintegration. Most of these complications can be prevented by filling the bone defect with materials that can replace the bone immediately after surgery and enhance the healing speed by stimulating new bone creation. Studies focused on replacement of the damaged tissues using autologous and allogeneic transplantation revealed considerable limitations and complication $(3,5,7,13)$. However, autografts are considered as gold standard of the treatment they are connected with needs of harvesting the bone from other bone source and therefore leads to another surgery, pain, scaring and possible complication during healing. Allografts on the other hand are connected with some ethical issues, many patient are not accepting tissue from the cadaver. Obtaining such a tissue is expensive and represents the risk of immunoreactions and transmission of infection. Nowadays, stem cell therapy and tissue engineering are commonly considered as new potential methods for the treatment of different types of diseases. It seems to be logical to combine MSCs together with osteoinductive scaffolds to accelerate bone healing. However, vascularization is still an issue that must be addressed (2). Even though stem cells are generally more resistant to hypoxia and low nutrition supply than somatic cells, we must ensure that the growth of the blood vessels is complete before maturation occurs.

\section{RESEARCH AIMS}

While most of the published studies take at least one month, but mostly 3-6 months to prove the effect of the BTE, the presented study is focused on early stage healing and the changes within the tricalcium phosphate scaffold loaded with human MSC. Moreover we tried to identify the differences resulting from usage of non-differentiated and pre-differentiated MSC.

Our research aims are, successively:

I. Confirm the survival of the MSCs loaded into the scaffold under in vivo conditions and the changes in the surrounding area during early stage healing (2 weeks).

II. Histologically evaluate the angiogenesis and new bone creation during early stage healing

III. Evaluate the benefits resulting from usage of the pre-differentiated MSCs

\section{DELIMITATION AND AREA OF THE STUDY}

To reach the research aims we compare the scaffold loaded with pre-differentiated, non-differentiated MSCs and scaffold which was not loaded with the MSCs applying the approach suggested by Rosset et al. (10). A group of rats was implanted with a bone scaffold loaded with pre-differentiated osteoblasts and pre-differentiated endothelial cells, and this group was compared to a group of rats implanted with the bone scaffold loaded with undifferentiated MSCs and group of rat implanted with the scaffold alone. To observe the early stage healing and new vessels creation the samples were taken back after 2 weeks in vivo and histologically evaluated. Results were compared in terms of immune reaction, new vessel formation and new bone formation.

\section{METHODS}

\section{CELL PREPARATION}

MSCs isolation was performed in GMP facility of Bioinova, Ltd. (Prague, Czech Republic). Adherent cells were cultured at $37^{\circ} \mathrm{C}$ in a humidified atmosphere containing $5 \%$ $\mathrm{CO}_{2}$ in enriched MEM Alpha media (Lonza, Walkersville, Maryland, USA) containing platelet lysate (5\%; Bioinova) and gentamycine $\left(10 \mu \mathrm{g} / \mathrm{ml}\right.$; Gentamicine Lek ${ }^{\circledR}$; Lek Pharmaceuticals, Ljublanja, Slovenia). The media was changed twice a week. According to their surface markers expression (Figure 1), spindle-shaped morphology and plastic adherence (Figure 2a) the cells were identified as MSCs. After reaching near-confluency, cells were harvested by a TrypLE $^{\text {TM }}$ (Life Technologies, Carlsbad, California, USA), passaged and seeded again onto a fresh plastic surface. Cells in the 2nd passage were allowed to differentiate into osteoblasts and endothelial cells according to standard differentiation protocols. Seven days after, pre-differentiated cells were harvested and seeded onto a synthetic bone

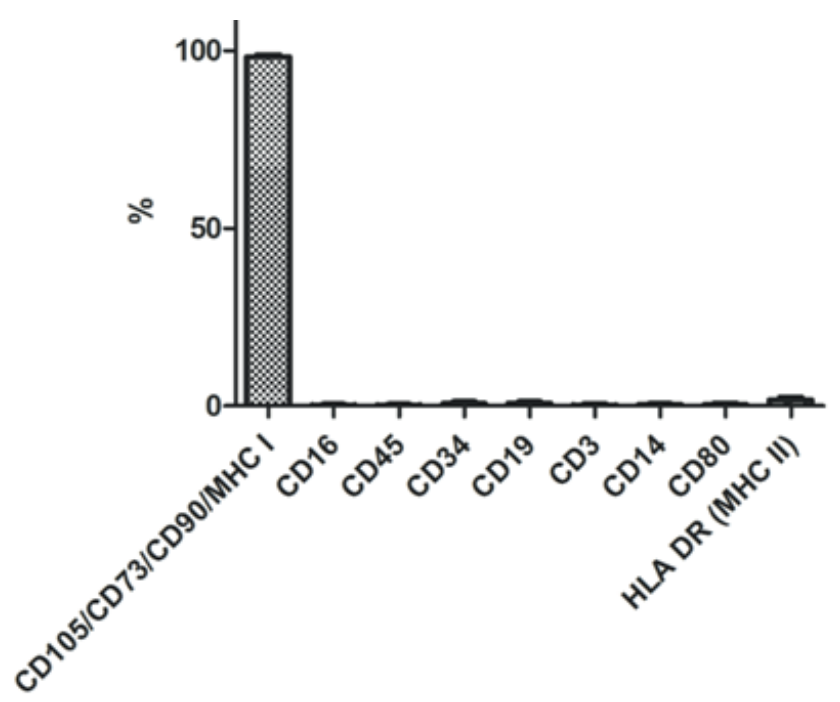

Fig. 1: Characterization of the MSCs in Suspension of autologous MSC 3P in $1.5 \mathrm{ml}$ (Bioinova). Expression of MSCs surface markers was analyzed on fluorescence-activated cell sorting (FACS) using antigens against these surface markers: CD105, CD73, CD90, $\mathrm{MHCl}$, CD16, CD45, CD34, CD19, CD3, CD14, CD80, HLA DR (MHC II). 
scaffold. Undifferentiated cells in the 3rd passage were also harvested and seeded onto a synthetic bone scaffold.

\section{OSTEOGENIC DIFFERENTIATION}

MSCs were seeded onto a $75 \mathrm{~cm}^{2}$ cultivation flask at a density of $3 \times 10^{3}$ cells $/ \mathrm{cm}^{2}$. The next day, cells were treated with a media consisting of MEM Alpha, $10 \% \mathrm{FBS}, 1 \% \mathrm{P} / \mathrm{S}^{\mathrm{TM}}$, $0.1 \mu \mathrm{M}$ dexamethasone, $10 \mathrm{mM} \beta$-glycerolphoshate and $0.1 \mathrm{mM}$ L-ascorbic acid (all from Sigma-Aldrich, St Louis, Missouri, USA). After 2 weeks in the culture, the cells were harvested and a control sample was fixed and stained with Alizarin Red $\mathrm{S}$ to detect calcium-rich deposits shown in Figure 2b.

\section{ENDOTHELIAL DIFFERENTIATION}

MSCs were seeded onto a $75 \mathrm{~cm}^{2}$ cultivation flask coated at a density of $2 \times 10^{3}$ cells $/ \mathrm{cm}^{2}$. Cells were cultivated in media consisting of MEM Alpha, $10 \% \mathrm{FBS}, 1 \% \mathrm{P} / \mathrm{S}^{\mathrm{TM}}, 50 \mathrm{ng} / \mathrm{ml}$ vascular endothelial growth factor (VEGF; Sigma, St Louis, Missouri, USA) and $10 \mathrm{ng} / \mathrm{ml}$ basic fibroblast growth factor (bFGF, Sigma, St Louis, Missouri, USA). After 2 weeks, the cells were harvested and a control sample was counterstained with DAPI and von Willebrand's factor antibody (Sigma, St Louis, Missouri, USA) to detect endothelial-like cells shown in Figure 2c.

\section{SYNTHETIC BONE SCAFFOLD}

PORESORB ${ }^{-}$-TCP (Lasak Ltd, Prague, Czech Republic) was used as a synthetic bone scaffold. This material is based on tricalcium phosphate and its structure is similar to that of bone, possessing two main sizes of porosity: macro-pores, approximately $100-200 \mu \mathrm{m}$ in size, and micro pores, ranging from 1 to $5 \mu \mathrm{m}$.

\section{ANIMALS}

Wistar rats (Velaz, Prague, Czech Republic) with body weights ranging from 550-600 g were used in this study. All experiments were done in compliance with the European Communities Council Directive of 24 November 1986 (86/609/EEC) regarding the use of animals in research and were approved by the Ethical Committee of the Institute of Experimental Medicine ASCR, Prague, Czech Republic. All efforts were made to minimize the number of animals used in the study. Rats were randomly divided into one of the following groups shown in Table 1: (i) rats implanted with $0.2 \mathrm{~g}$ of synthetic bone scaffold only (group $1 ; \mathrm{n}=14$ ), (ii) rats implanted with $0.2 \mathrm{~g}$ of synthetic bone scaffold
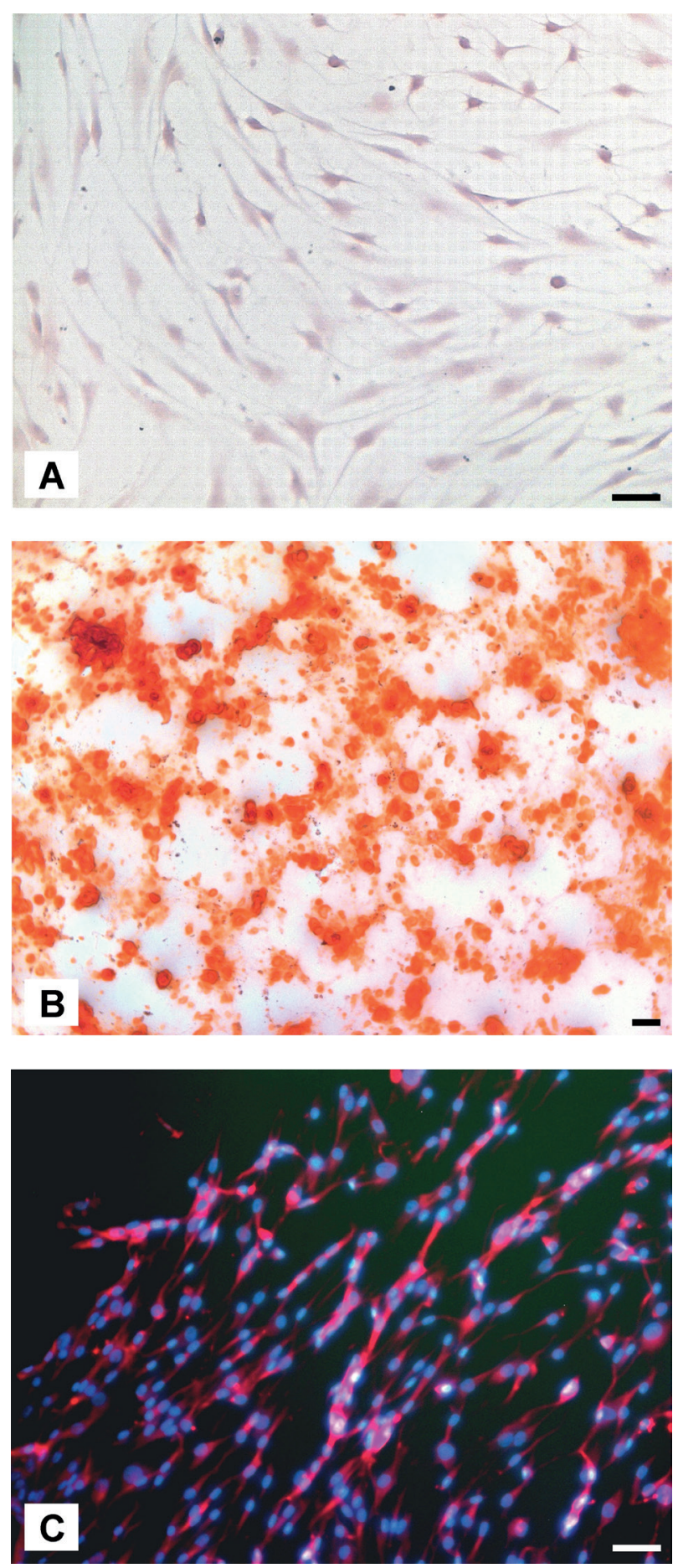

Fig. 2: Ilustration of undifferentiated MSCs stained with hematoxylin-eosinofil ( $A$, original magnification 100x); predifferentiated osteoblasts stained with Alizarin Red S (B, original magnification 50x); pre-differentiated endothelial cells stained with DAPI and von Willebrand's factor antibody (C, original magnification $200 \times$ ).

\begin{tabular}{l|l|l|l}
\hline Group & Number of rats & Implanted material & Type and amount of MSCs \\
\hline 1 & 14 & Poresorb $^{\circledR}-\mathrm{TCP}$ & - \\
\hline 2 & 7 & Poresorb $^{\circledR}-\mathrm{TCP}$ & $2 \times 10^{6}$ undifferentiated MSCs \\
\hline 3 & 7 & Poresorb $^{\circledR}-\mathrm{TCP}$ & $\begin{array}{l}10^{6} \text { pre-differentiated osteoblasts }+10^{6} \text { pre-differentiated } \\
\text { endothelial-like cells }\end{array}$ \\
\hline
\end{tabular}

Tab. 1: Characteristics of animal groups implanted with combined biomaterial based on tricalcium phosphate and mesenchymal stromal cells (MSCs). 
seeded with $2 \times 10^{6}$ MSCs (group 2; $\mathrm{n}=7$ ), and (iii) rats implanted with $0.2 \mathrm{~g}$ of synthetic bone scaffold seeded with $1 \times 10^{6}$ pre-differentiated osteoblasts and $1 \times 10^{6}$ pre-differentiated endothelial cells (group 3; $\mathrm{n}=7$ ).

\section{SURGERY}

After the induction of anesthesia, using $5 \%$ isoflurane in room air (flow $300 \mathrm{~mL} / \mathrm{min}$ ), the animals were maintained in $2 \%$ isoflurane anesthesia (flow $300 \mathrm{~mL} / \mathrm{min}$ ) via a face mask throughout the operation. $0.1 \mathrm{ml}$ of analgesic Rymadile (Pfizer Animal Health SA, Louvain-la-Neuve, Belgium) was injected intramuscularly. Under aseptic conditions, a $2 \mathrm{~cm}$ lateral skin incision was performed on the back of the animal. The osteogenic biomaterial was implanted into the intramuscular pocket. The soft tissue and skin was sutured with non-resorbable thread. Transplanted animals were immunosuppressed on a daily basis with $10 \mathrm{mg} / \mathrm{kg}$ cyclosporine (Sandimmun ${ }^{\circledR}$, Novartis, Basel, Switzerland) administered intraperitoneally; this procedure started from the day before surgery and lasted until euthanasia. Bacterial infection was prevented by an intramuscular gentamycin injection $(60 \mathrm{mg} / \mathrm{kg}$; Gentamicine Lek ${ }^{\oplus}$; Lek Pharmaceuticals, Ljublanja, Slovenia). The rats were euthanized 14 days after surgery.

\section{HISTOLOGICAL ANALYSIS}

At the end of the experiment, the animals were intracardially perfused under deep anesthesia (pentobarbital 150 $\mathrm{mg} / \mathrm{kg}$ ) with $4 \%$ formaldehyde in 0.1 M PBS (IKEM, Prague, Czech Republic). The tissue containing implants were dissected from the area, postfixed in $10 \%$ formaldehyde and further decalcified with formic acid (Fingerland's department of Pathology, Hradec Králové, Czech Republic). One paraffin block was prepared from each sample, cut into $4 \mu \mathrm{m}$ thin sections and stained with hematoxylin-eosin (H\&E). Sections were examined under a light microscope and histomorphometrical analysis was performed using NIS-Elements software (Nikon Instruments, Inc., USA). Immunofluorescent staining for DAPI (Sigma, St Louis, Missouri, USA), RECA, COLL1, MT-CO2 (all from Abcam, Princeton, New Jersey, USA) was used to identify the potential survival of transplanted cells. Antigen-antibody complexes were visualized using goat anti-mouse IgG secondary antibody conjugated with Alexa-Fluor 488 (Molecular Probes Invitrogen, Carlsbad, California, USA). The samples were examined using a spectral confocal microscope (Carl-Zeiss, Oberkochen, Germany). Results were calculated as a percentage of positive samples in the whole sample group.

\section{RESULTS}

All animals were successfully implanted with the osteoconductive biomaterial without rejection. Hematoxylin-eosin staining revealed deposits of calcified material surrounded by multinucleated foreign body giant cells in all three groups visualized in Figure 3a. Neither neutrophils, nor lymphocytes were present, implying that there was no inflammation caused by implantation of the combined biomaterial or of the separate osteoconductive biomaterial, respectively (visualized in Figure 3b). However, no new bone or cartilage formation was detected by hematoxylin-eosin staining. In the vicinity of the implant, signs of skeletal muscle regeneration associated with surgery were evident according to small profiles of myotubes with centrally located nuclei.

Immunohistochemical analysis confirmed the biocompatibility of the human MSCs with the calcium triphosphate synthetic bone scaffold. MSCs were detected by positive DAPI and MTCO2 staining in $43 \%$ of all the samples implanted with MSCs, in $57 \%$ of samples with undifferentiated MSCs (group 2) and in $28 \%$ of samples with pre-differentiated MSCs (group 3 ) as shown in Table 2 and Figure 4a. MSC survival rate was not affected by differentiation. Positive RECA staining was found in $93 \%$ of all samples implanted with MSCs. No significant differences were found between group 3 , which involved implantation with pre-differentiated MSCs $(100 \%$ of samples stained positively), and group 2 with undifferentiated MSCs ( $86 \%$ of samples stained positively), as shown in Table 2 and in Figure $4 \mathrm{~b}$.

Results from the histological evaluation shown in Figure $4 \mathrm{c}$ were confirmed by COLL1 staining. Positive COLL1 staining was detected in only three samples, all of which were in group 2 and had been implanted with undifferentiated MSCs.

\begin{tabular}{l|c|c|c} 
& MTCO2 & RECA & COLL1 \\
\hline Group 1 & $0 / 14$ & $0 / 14$ & $0 / 14$ \\
\hline Group 2 & $4 / 7$ & $6 / 7$ & $3 / 7$ \\
\hline Group 3 & $2 / 7$ & $7 / 7$ & $0 / 7$ \\
\hline
\end{tabular}

Tab. 2: Immunohistochemical analysis of tissue sections. Group 1 $(n=14)$ represents control animals implanted with PORESORB $^{\circledR}$-TCP; group $2(n=7)$ represents animals implanted with PORESORB ${ }^{\circledR}-$ TCP seeded with mesenchymal stromal cells (MSCs); group $3(n=7)$ represents animals implanted with PORESORB ${ }^{\circledR}$-TCP seeded with pre-differentiated osteoblasts and endothelial-like cells; all groups stained with MTCO2, RECA and COLL1. Data are presented as the number of positive stained sections in the whole sample group.

\section{DISCUSSION}

It has been established, that bone tissue engineering (BTE) using combinations of scaffold loaded with the cells is more effective than usage of scaffold or cells alone (1).

According to results of the presented study we proved that both predifferentiated and non-differentiated cells were able to survive within the scaffold for 14 days. But survival rate of the non-differentiated MSC were twice higher. Positive staining for human cells DNA and MTCO2 was positive in $57 \%$ slides acquired from the scaffolds loaded with undifferentiated MSC compared to $28 \%$ slides acquired from the scaffolds loaded with pre-differentiated MSC. As expected we haven't identified any newly created bone tissue within the samples of non-differentiated MSC group but surprisingly neither within the samples 
used predifferentiated MSC, even though we proved the ability of osteoid matrix creation during in vitro study. We believed that this is the consequence of hypoxia and low nutrients supply. On the other side we were able to indentify newly created vessels in all samples so we can predict that the proliferation, differentiation and creation of the bone tissue will start in the next phase of the regeneration. From above written we conclude that using the predifferentiated stem cells do not lead to the better results, but even more due to the lowered survival rate it can leads to delayed healing.

These finding are in contradiction with many other studies $(12,15)$ who reported that pre-differentiation of MSCs with inductive factors prior to transplantation may enhance the differentiation response and therefore enhance the healing process. This discrepancy can be explained by using different scaffold material, different size of scaffold, or more likely by using inductive factors within the scaffold. On the other side our finding supports the theory of Gronthos et al. (8) who presented that the survival of human MSCs depends on the immaturity of the cells, thus some applications may possibly require minimizing the maturation of MSCs. Shimizu et al. (11) observed the newly created bone 14 days after implantation, but compare to our study they used MSC pre-differentiated for 4 weeks and the cells were not seeded within the scaffold but in the multilayered sheet-like structure.

Our study supports Amini (2) who classify the vascularization as the scaffold between the fundamental challenges of BTE. We have to take in mind that most of the in vivo studies (including our study) are done in the place where the scaffolds are surrounded by the soft tissue and not within the bone which will offer even lower chance for vascularization. How to deal with this challenging task? Vascularization depends on many different factor. First of all the scaffold porosity is crucial. In the study of Kara- georgiou and Kaplan published in 2005 (9) was recommended to use at least $300 \mu \mathrm{m}$ pores within the scaffold. Neo vascularization can be enhancing by the inclusion of the angiogenic growth factors (VEGF, PDGF and bFGF). The pros of this way are: easiness, possibility of controlled and prolonged releasing, but the cons are: high price and in case of not well balanced concentration the newly created vessels can lead to vascular leakage demonstrated by Zisch et al. (14). The last option is pre-vascularizations of the scaffold before implantation. This process can be done under in vivo or in vitro conditions. For in-vitro pre-vascularization is recommended to co-culture the scaffold with the endothelial and osteogenic cells before implantation. This leads to creation of immature vessels which will mature and connect with the recipient vessels after implantation. This method leads to increased bone formation in vivo $(1,4,6)$. The second option is based upon the implantation of the scaffold within the well vascularized tissue. This will lead to ingrowth of the vessels into the scaffold and creation of mature arteries and veins. The pros of this procedure is the possibility to connect the scaffold vessel system directly to the recipient blood circulation using microsurgical and therefore obtained immediate blood perfusion. The cons consist in the long time preparation (the whole procedure takes several weeks - depending on the vascularization of the surrounding tissue), need of another surgical procedure (2).

\section{CONCLUSION}

Cell therapy is a perspective therapeutic approach in the treatment of bone defects and management of bone cysts. MSCs were compatible with the commercially available bone scaffold tricalcium phophate and human MSCs were detected inside the implants 14 days after transplantation.
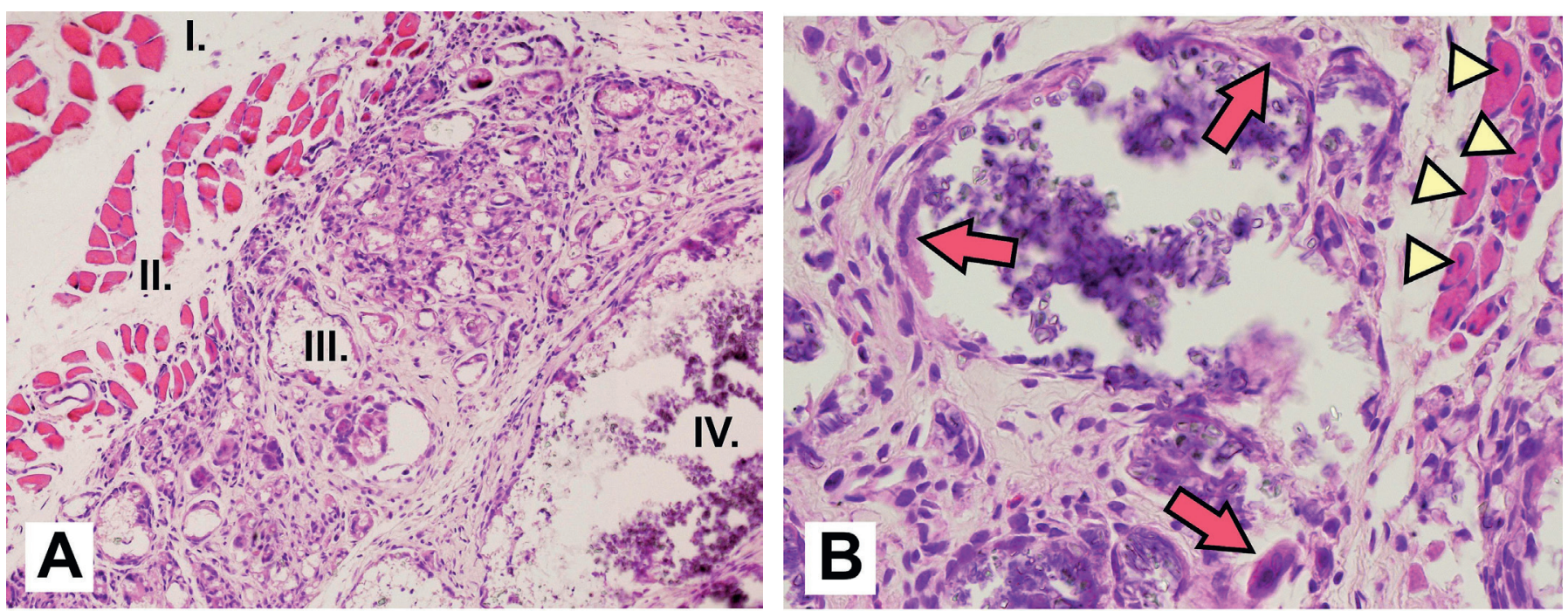

Fig. 3: Histological analysis of implant sections stained with hematoxylin eosin. A) Border area shows a stratification of reactions toward the implant. Zone I contains intact extrafusal skeletal muscle, zone II contains a muscle injured during implantation with signs of regeneration. The peripheral zone of the implanted material (zone III) is intermingled with degenerating muscle whereas the inner area (IV) shows the reaction of the host tissue to the implant. B) A detailed micrograph indicates the formation of giant multinucleated bodies at the surface of the implant (red arrows). Regenerating myotubes with centrally located nuclei are marked with yellow arrowheads (original magnification 400×). 
This study showed no significant differences between using pre-differentiated osteoblasts combined with pre-differentiated endothelial cells in the bone scaffold and undifferentiated MSCs in the bone scaffold in terms of immune reaction, new vessel formation and bone formation. However, 14 days seem to be a short time for new bone formation assessment.
ACKNOWLEDGEMENTS

Supported by the PRVOUK P37/13 and PRVOUK P37/06.

\section{CONFLICTS OF INTEREST}

The authors declare that they have no conflicts of interests concerning this article.
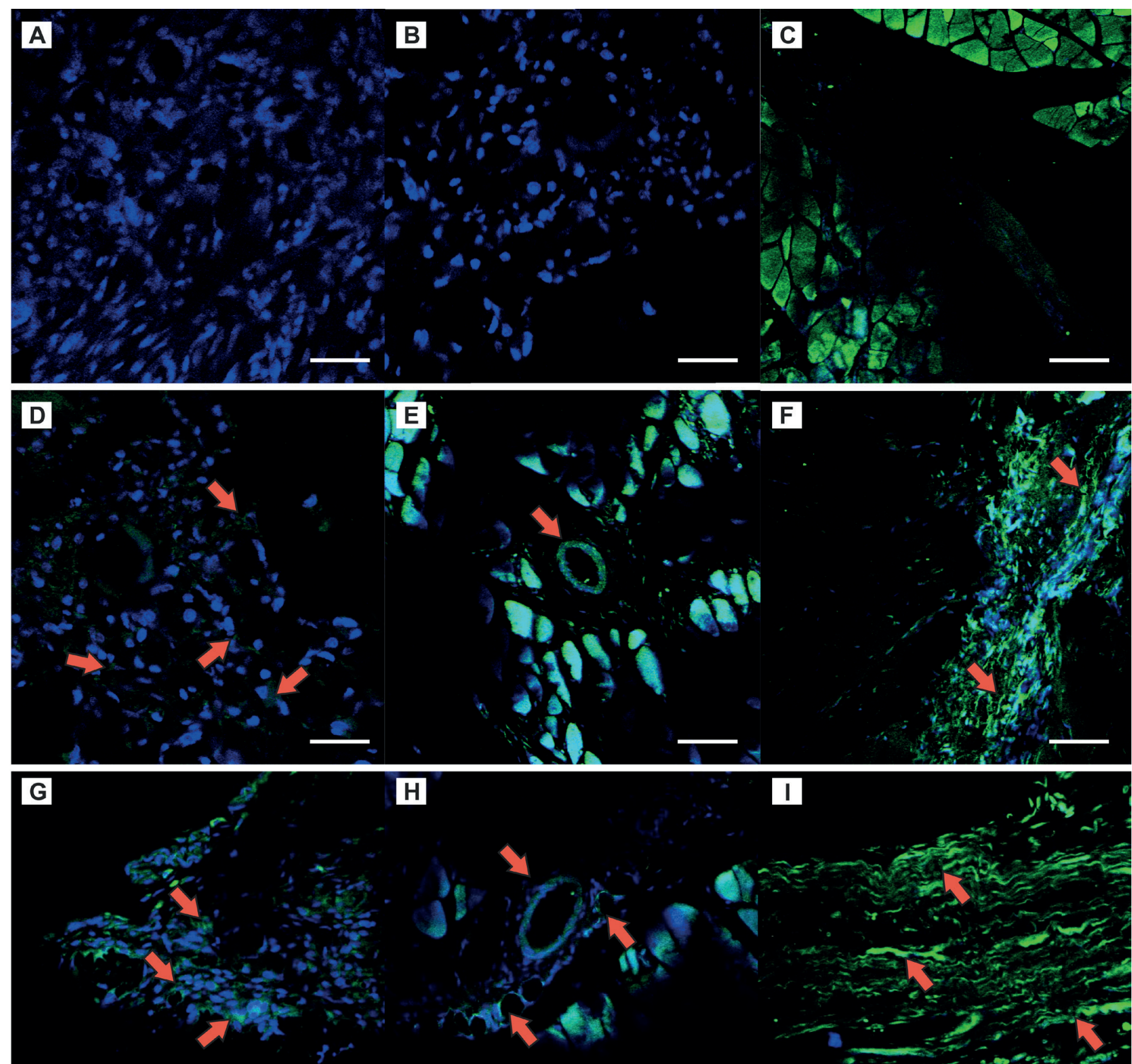

I

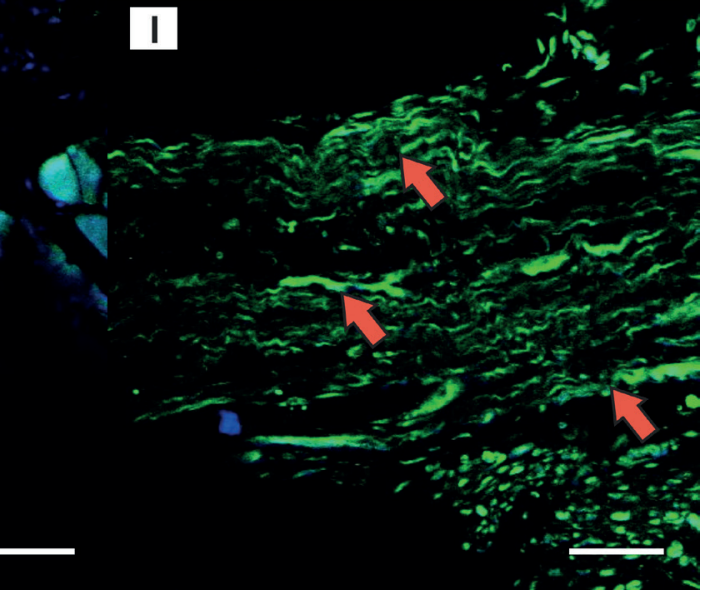

Fig. 4: Immunohistochemical analysis of implant sections. In rows sections from group $1(n=14)$, group $2(n=7)$ and group $3(n=7)$. Sections stained with DAPI and MTCO2 (A; D; G); arrows show mitochondria inside the cells of human origin. Sections stained with DAPI and RECA (B; E; H); arrows show endothelial cells $(C ; F ; I)$. Arrows show positive COLL1 staining for detection of collagen type I of newly formed bone. Bars show $50 \mu \mathrm{m}$. 


\section{REFERENCES}

1. Amini AR, Laurencin CT, Nukavarapu SP. Bone tissue engineering: recent advances and challenges. Crit Rev Biomed Eng 2012; 40: $363-408$.

2. Amini AR, Laurencin CT, Nukavarapu SP. Differential analysis of peripheral blood- and bone marrow-derived endothelial progenitor cells for enhanced vascularization in bone tissue engineering. J Orthop Res 2012; 30: 1507-1515.

3. Baroli B. From natural bone grafts to tissue engineering therapeutics: brainstorming on pharmaceutical formulative requirements and challenges. J Pharm Sci 2009; 98: 1317-1375.

4. Buschmann J, Welti M, Hemmi S, Neuenschwander P, Baltes C, Giovanoli P, Rudin M, Calcagni M. Three-dimensional co-cultures of osteoblasts and endothelial cells in DegraPol foam: histological and high-field magnetic resonance imaging analyses of pre-engineered capillary networks in bone grafts. Tissue Eng Pt A 2010; 17: 291299.

5. Dimitriou R, Jones E, McGonagle D, Giannoudis PV. Bone regeneration: current concepts and future directions. BMC Med 2011; 9: 66.

6. Fedorovich NE, Haverslag RT, Dhert WJ, Alblas J. The role of endothelial progenitor cells in prevascularized bone tissue engineering: development of heterogeneous constructs. Tissue Eng Pt A 2010; 16: 2355-2367.

7. Gazdag AR, Lane JM, Glaser D, Forster RA. Alternatives to autoge- nous bone graft: efficacy and indications. J Am Acad Orthop Sur 1995; 3: $1-8$.

8. Gronthos S, Chen S, Wang CY, Robey PG, Shi S. Telomerase accelerates osteogenesis of bone marrow stromal stem cells by upregulation of CBFA1, osterix, and osteocalcin. J Bone Miner Res 2003; 18: 716-22.

9. Karageorgiou V, Kaplan D. Porosity of 3D biomaterial scaffolds and osteogenesis. Biomaterials 2005; 26: 5474-5491.

10. Rosset P, Deschaseaux F, Layrolle P. Cell therapy for bone repair. Orthop Traumatol Surg Res 2014; 100: 107-112.

11. Shimizu K, Ito A, Yoshida T, Yamada Y, Ueda M, Honda H. Bone tissue engineering with human mesenchymal stem cell sheets constructed using magnetite nanoparticles and magnetic force. J Biomed Mater Res B 2007; 82: 471-480.

12. Schliephake H, Knebel JW, Aufderheide M, Tauscher M. Use of cultivated osteoprogenitor cells to increase bone formation in segmental mandibular defects: an experimental pilot study in sheep. Int J Oral Max Surg 2001; 30: 531-537.

13. Soucacos PN, Johnson EO, Babis G. An update on recent advances in bone regeneration. Injury 2008; 39: 1-4.

14. Zisch AH, Lutolf MP, Hubbell JA. Biopolymeric delivery matrices for angiogenic growth factors. Cardiovasc Pathol 2003; 12: 295-310.

15. Zhou G, Liu W, Cui L, Wang X, Liu T, Cao Y. Repair of porcine articular osteochondral defects in non-weightbearing areas with autologous bone marrow stromal cells. Tissue Eng 2006; 12: 3209-3221. 